$\underline{\text { Preprint typeset in JHEP style - HYPER VERSION }}$

SLAC-PUB-10094

\title{
Yukawa Hierarchies From Extra Dimensions With Small FCNC
}

\author{
Ben Lillie \\ Stanford Linear Accelerator Center \\ Stanford CA 94309, USA \\ iillieb@slac.stanford.edu*
}

\begin{abstract}
We investigate a class of extra dimensional models where all of the Standard Model fermions are localized to a single fixed point in an $S_{1} / Z_{2}$ orbifold, and each species is localized with an exponential wavefunction with a different width. We show that this naturally generates Yukawa hierarchies of the size present in the Standard Model, and we find a set of model parameters that reproduces the observed masses and mixings to experimental accuracy. In addition, the dominant constraints, arising from flavor changing neutral currents, are shown to restrict the compactification scale to be $1 / R \geq 2-5 \mathrm{TeV}$, which is a much less stringent constraint than in similar extra dimensional models of the Yukawa hierarchy.
\end{abstract}

Keywords: Quark Masses, Compactification, Beyond the Standard Model.

${ }^{*}$ Work supported by the Department of Energy, Contract DE-AC03-76SF00515 


\section{Contents}

1. Introduction 1

2. Yukawa Hierarchies 2

3. Standard Model parameters 6

4. Flavor Changing Processes 8

5. Discussion

6. Conclusion

\section{Introduction}

Recently there has been much interest in the possibility that there exist extra dimensions much larger than the Planck scale [1, 2, 3, 4, 5, 6]. There are many reasons for this excitement, particularly the fact that having large dimensions accessible to gravity may allow a solution of the hierarchy problem associated with the Higgs mass [7, 8, 9, 10, 11]. However, extra dimensions may also be able to address other puzzles left unexplained in the Standard Model, such as why there are three families [12], or the origin of dark matter [13, 14, 15, 16].

In particular, it was noticed by Arkani-Hamed and Schmaltz (AS) that localizing the standard model fermion fields in an extra dimension could solve the other serious hierarchy problem, that of the relative size of the fermion masses [17]. This is accomplished by assigning a universal Yukawa coupling in the higher dimensional theory, but separating the left and right handed components of the effective $4 \mathrm{D}$ fermions in the additional dimension. This is accomplished by localizing the zero modes to Gaussian profiles, and separating the centers of the Gaussians. The 4D Yukawa couplings, and hence fermion masses, are proportional to integrals of products of these zero modes over the compact dimension. They are thus suppressed by exponentially small wavefunction overlaps. Realizations of this model that match the Standard Model masses and mixing parameters have been produced [18, 19, 20, 21, 22, 23]. There also exist possibilities for 
observing the effects of this localization at future colliders, such as the production of Kaluza-Klein excitations of the Standard Model particles. Current results from the direct production of KK states. and precision electro-weak measurements restrict the size of dimensions accessible to Standard Model fields to be $R \lesssim$ few $\mathrm{TeV}^{-1}$ [24, 25, 26, 27]. The separation between fields also allows for detection of some novel effects 228, 29.

However, split fermion models also generate tree-level flavor-changing neutral currents (FCNC). Strict bounds from these are hard to obtain due to the large number of model parameters, but in general one finds $R \lesssim 400 \mathrm{TeV}^{-1}$ [20, 30, 31, 32]. Without miraculous cancellations, this can be evaded only by making very small the ratio $\rho=\sigma / R$, where $\sigma$ is the width of the localized fermions, thus introducing a new hierarchy.

In this paper we present a different model based on similar ideas. Instead of fixed width fermions localized at different points, we consider variable width fermions localized at the same point. We will show that this model can produce the observed fermion mass hierarchy and mixing angles, including the CP violating phase while generating much smaller FCNC. This model has the additional virtue suppressing any proton decay operators. The resulting constraints on the compactification scale turn out to be a factor of 30 smaller than similar constraints on the AS model, and can be as low as $1 / R \geq 2 \mathrm{TeV}$. This is low enough to raise the exciting possibility that they could be within reach of the next generation of colliders, and that the model could be embedded in a more encompassing one that also solves the Higgs mass hierarchy problem. This is similar to the proposal of Kaplan and Tait [22], although they still required the left and right handed fermions to be separated from each other. This idea is also similar to a model studied in the case of a warped extra dimension [33.

This paper is organized as follows. In section 2 we show how variable widths can explain the Yukawa hierarchy. A specific set of model parameters that reproduce the observed fermion masses and mixing angles is shown in section 3 . In section 4 we consider the constraints from FCNC. Section 5 discusses possible mechanisms for implementing the model, and section 6 concludes.

\section{Yukawa Hierarchies}

We propose the configuration where different fermion flavors are localized in the extradimensional bulk as exponentially localized wavefunctions with different widths, as illustrated in Fig. 1. For most of this paper we consider this to be a phenomenological ansätz. A discussion of what types of models may produce this picture is given in section 5. This paper will focus on models with a single compact extra dimension, 


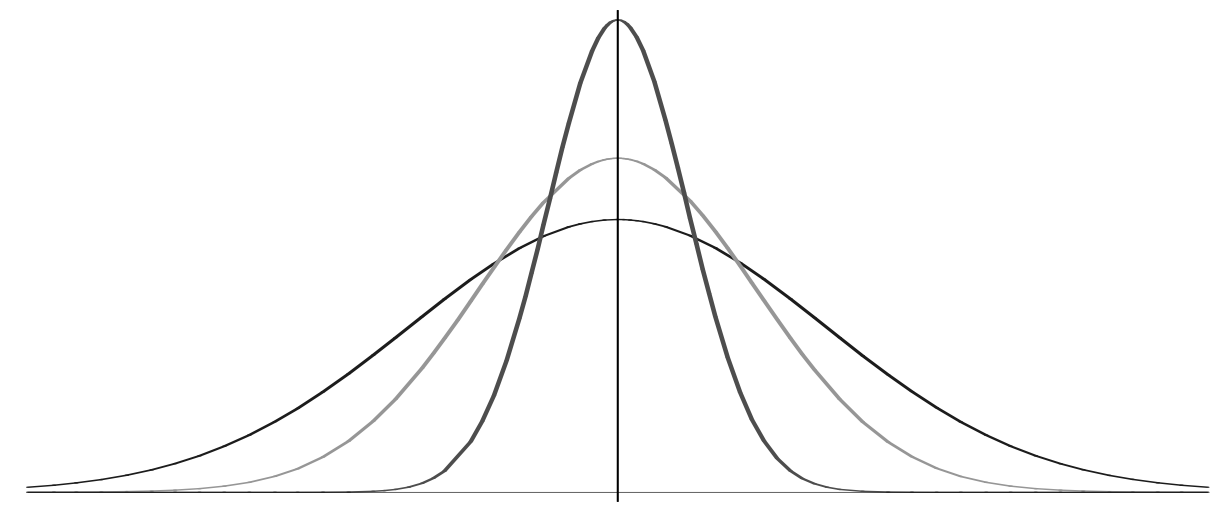

Figure 1: Illustration of the idea of variable width fermions localized to a single point.

which we take to be the interval $[0, R]$, with the edges of the additional dimension being defined either by a brane configuration or by an orbifolding condition.

We begin with a general configuration that allows each fermion species to be localized with different widths to a specific point. A fermion is taken to be localized at $x_{0}$ with width $\sigma$ if it has a profile $\psi \propto e^{-\left|x-x_{0}\right|^{a} / \sigma^{a}}$. The power $a$ is determined by the localization mechanism, and represents a phenomenological parameterization of the unknown mechanism. In the AS model, for instance, fermions are localized with Gaussian profiles, so $a=2$; in the Randall-Sundrum (RS) scenario, or in brane-worlds with bulk mass terms, fermions are localized to exponentials, so $a=1$. For simplicity, we take the localization point to be $x_{0}=0$. The normalized wavefunction for the $i$-th fermion is

$$
\psi_{i}=\frac{2^{1 / 2 a}}{\sqrt{\sigma_{i}}} \frac{1}{\sqrt{\alpha_{a}(R, \sigma)}} e^{-y^{a} / \sigma_{i}^{a}}
$$

where we have defined

$$
\alpha_{a}(R, \sigma)=\int_{0}^{\frac{R}{\sigma}} d z e^{-z^{a}} .
$$

This integral will cancel to good approximation in the following computations of the coupling constants.

The gauge boson wavefunctions will also generally depend on the model details. For the moment the only important point is that the Higgs zero mode be flat in the extra dimension, so $h^{(0)}(y)=1 / \sqrt{R}$, where $h$ is the Higgs field and $h^{(0)}$ is the zero mode of the Kaluza-Klein expansion. This zero mode corresponds to the Standard Model Higgs, the vev of which is responsible for electro-weak symmetry breaking and generating 4D 
fermion masses. The 5D Yukawa couplings for the fermions carry mass dimension $-1 / 2$. In the dimensionally reduced theory this scale is given by the compactification scale $R$. We therefore write the 5D Yukawa terms as

$$
\mathcal{L}_{\text {Yukawa }}^{5 D}=\lambda_{5, i j} \sqrt{R} h \bar{\psi}_{i} \psi_{j}
$$

The dimensionally reduced 4D Lagrangian is obtained by integrating over the compact dimension. The 4D Yukawa couplings are then given, in the physically appropriate approximation that $R$ is much larger than any of the widths, by

$$
\begin{aligned}
\lambda_{4, i j} & =\lambda_{5, i j} \sqrt{R} \int_{0}^{R} d y h^{(0)}(y) \bar{\psi}_{i}(y) \psi_{j}(y) \\
& =\lambda_{5, i j} \sqrt{R}\left(\frac{2^{1 / a} \sqrt{\sigma_{i} \sigma_{j}}}{\left(\sigma_{i}^{a}+\sigma_{j}^{a}\right)^{1 / a}}\right) .
\end{aligned}
$$

This can be rewritten as

$$
\lambda_{4, i j}=\lambda_{5, i j} \sqrt{R}\left(2^{1 / a}\left(\left(\frac{\sigma_{i}}{\sigma_{j}}\right)^{a / 2}+\left(\frac{\sigma_{j}}{\sigma_{i}}\right)^{a / 2}\right)^{-1 / a}\right) .
$$

The factor in parenthesis is determined by the wavefunction overlap. It is unity when $\sigma_{i}=\sigma_{j}$, and does not change dramatically as the widths vary. If we take all the 5D Yukawa couplings to be the same, $\lambda_{5, i j}=\lambda_{5}$ we expect this model to have 4D Yukawa matrix elements given by $\lambda_{4, i j} \approx \lambda_{5}$, and essentially equal with small differences due to the different widths. This is a realization of the "democratic" scenario of fermion mass generation [34. This scenario relies on the observation of the singular values of the matrix

$$
A=\left(\begin{array}{lll}
1 & 1 & 1 \\
1 & 1 & 1 \\
1 & 1 & 1
\end{array}\right) \stackrel{\operatorname{diag}}{\longrightarrow}\left(\begin{array}{lll}
3 & 0 & 0 \\
0 & 0 & 0 \\
0 & 0 & 0
\end{array}\right)
$$

That is, there is one large singular value and two vanishing ones. If one then perturbs the values of the elements in $A$, the two zero diagonal values become finite, but small. When $A$ is a Yukawa matrix this gives a natural hierarchy in the resulting masses. This is in stark contrast to the AS scenario, where the hierarchy is generated by small elements directly in the Yukawa matrices.

We have studied the expected size of the resulting Yukawa hierarchy by performing random trials. We drew six widths for the 5D fermion wavefunctions from the interval $[1,3]$ and computed the physical mass spectrum for the case $a=2$. The interval 


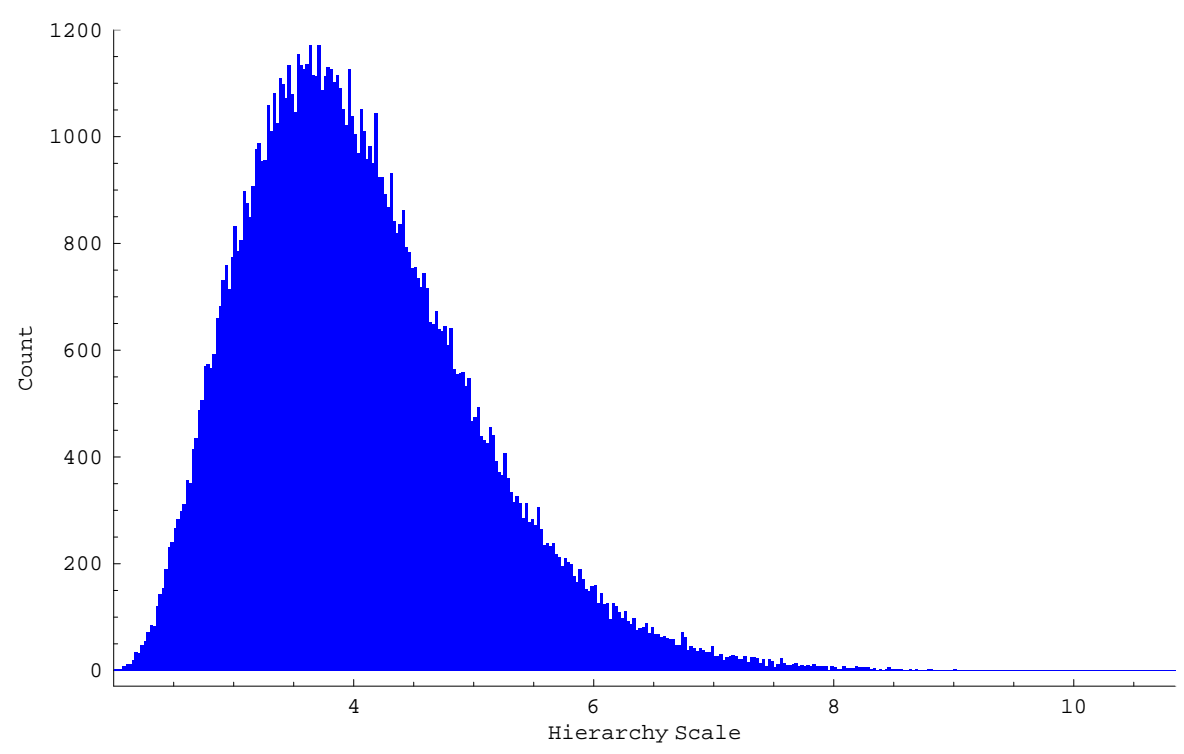

Figure 2: Histogram of the expected size of the Yukawa hierarchy for $10^{5}$ random configurations of fermion widths, with the widths drawn from the interval $[1,3]$. The hierarchy scale is defined as $\log _{10}\left(m_{1} / m_{3}\right)$, where $m_{1}\left(m_{3}\right)$ is the largest (smallest) mass resulting from that configuration.

was chosen so that all widths are of the same order of magnitude; the overall scale is irrelevant. The size of the hierarchy is taken to be $\log _{10}\left(m_{1} / m_{3}\right)$, where $m_{1}$ is the largest mass, and $m_{3}$ the smallest. Fig. 2 shows a histogram of the resulting hierarchies for $10^{5}$ random trials. We see that hierarchies of size 3 to 5 are generic.

It is important to note that Fig 2 shows the hierarchy between the masses resulting from a single Yukawa matrix; i.e., between fermions with the same quantum numbers. In this democratic scheme the largest singular value (and hence mass) is always of the same order of magnitude. Therefore it is impossible to generate the the large ratios $m_{t} / m_{b}$ or $m_{t} / m_{\tau}$ simply by varying the widths. We speculate on how to obtain these ratios within the model in section 5; for now we simply take the five dimensional Yukawa couplings $\lambda_{5}^{u}, \lambda_{5}^{d}, \lambda_{5}^{e}$ to be different.

Finally, note that while Fig. 2 was computed for $a=2$, similar results hold for any value of $a$. The important point is that the mass matrix is almost universal, and that small perturbations are generated by slightly different zero mode profiles. In addition, the Higgs profile need not be perfectly flat. As long as the variation in the Higgs 5D wavefunction is slow across the width of the fermions the same pattern will emerge. 


\section{Standard Model parameters}

For illustration, we will now obtain a set of model parameters that generates the observed fermion masses and mixings of the Standard Model to within experimental accuracy. For now, our model contains the following: There is the localization mechanism parameter $a$, which will be fixed to 2 in this section. There are the three Yukawa couplings $\lambda_{5}^{u}, \lambda_{5}^{d}, \lambda_{5}^{e}$. For the quark sector, there are nine widths, one each for the three left handed quark doublets, $Q_{i}$, the three right handed up singlets, $\bar{u}_{i}$, and the three right handed down singlets, $\bar{d}_{i}$. Only eight of the widths are independent because the Yukawa matrix elements from Eq. 2.4 only depend on the ratios of widths. For the lepton sector there are at least another six widths, for the doublets $L_{i}$, and the charged singlets $e_{i}^{+}$. There are possibly three more depending on whether right-handed neutrinos are included. For the purposes of this paper we ignore the neutrinos and only match the charged lepton masses to their observed values. Finally, it is necessary to include two arbitrary, but small, phases in one of the quark Yukawa matrices to be able to match all the CKM mixing angles and incorporate $\mathrm{CP}$ violation.

As a proof of principle, we have performed a search of the parameter space and located a configuration that reproduces the Standard Model parameters. For this search we used the values of quark masses and mixing angles from [35. The fermion masses were evaluated at the common scale $m_{t}$ using factors given in [19]. The values of the three five dimensional Yukawa couplings were obtained by matching to the large third generation masses (so $\lambda_{5}^{u}=\sqrt{2} m_{t} / v$, etc. where $v$ is the Higgs vev). The smaller fermion masses are then obtained by diagonalizing the Yukawa matrices generated from Eq. (2.4). The mixing angles can then be obtained from the relation

$$
V_{\mathrm{ckm}}=V_{L}^{(u) \dagger} V_{L}^{(d)}
$$

where the $V_{L}^{(u, d)}$ are the unitary matrices that rotate the left-handed $(u, d)$ quark fields to diagonalize the Yukawa matrices. The real part of the CKM matrix was parameterized by matching to the magnitude of the three entries above the diagonal, $V_{u s}, V_{u b}$, and $V_{c b}$. Finally, to match the observed CP phenomenology we included two arbitrary phases, $\phi_{1}$, in in $\lambda_{d d}^{(d)}$ and $\phi_{2}$ in $\lambda_{s s}^{(d)}$, and computed the Jarlskog invariant

$$
J=\operatorname{Im}\left(V_{u s} V_{c b} V_{u b}^{*} V_{c s}^{*}\right)
$$

and required it to be near the Standard Model expectation $J \approx 3 \times 10^{-5}$. The search was performed by Monte Carlo sampling of the parameter space to find a reasonably close match. 
In the lepton sector we are only matching three masses with five parameters, so solutions are essentially trivial to come by. For illustration, one such set is

$$
L_{i}=\left(\begin{array}{l}
6.118 \\
5.815 \\
2.360
\end{array}\right), \quad e^{+}=\left(\begin{array}{l}
2.696 \\
3.443 \\
5.576
\end{array}\right)
$$

which produces $m_{e}=0.511 \mathrm{MeV}$ and $m_{\mu}=105 \mathrm{MeV}$ from matching the coupling to $m_{\tau}=1777 \mathrm{MeV}$. There are many others that will match the leptonic masses just as well.

In the quark sector there are many more constraints. It is possible that there are many configurations that match the Standard Model, but since this search was for illustrative purposes only the search was stopped after a solution was obtained. This configuration is

$$
Q_{i}=\left(\begin{array}{l}
8.132 \\
2.365 \\
6.235
\end{array}\right), \quad \bar{u}=\left(\begin{array}{l}
9.865 \\
9.279 \\
9.404
\end{array}\right), \quad \bar{d}=\left(\begin{array}{l}
7.218 \\
6.463 \\
8.073
\end{array}\right), \quad \phi_{1}=-0.0140, \quad \phi_{2}=-0.0633
$$

These produce the values

$$
\begin{array}{cl}
m_{t}=175 \mathrm{GeV} & m_{b}=4.30 \mathrm{GeV} \\
m_{c}=1.31 \mathrm{GeV} & m_{s}=107 \mathrm{MeV} \\
m_{u}=2.00 \mathrm{MeV} & m_{d}=8.02 \mathrm{MeV}
\end{array}
$$

The absolute values of the resulting CKM matrix are

$$
\left|V_{\mathrm{CKM}}\right|=\left(\begin{array}{ccc}
0.975 & 0.224 & 0.00482 \\
0.224 & 0.974 & 0.0439 \\
0.00590 & 0.0438 & 0.999
\end{array}\right)
$$

The Jarslkog invariant is $J=2 \times 10^{-5}$. Another way of estimating the magnitude of $\mathrm{CP}$ violation is to calculate $\sin 2 \beta$. Doing this we find $\sin 2 \beta=0.627$, which is less than $2 \sigma$ away from the current world average $\sin 2 \beta=0.734 \pm 0.055$. 36 Interestingly, under the substitution $\phi_{1,2} \rightarrow-\phi_{1,2}$ all masses and absolute values of CKM elements remain the same, and $J$ and $\sin 2 \beta$ switch sign, picking up the alternate solution that results from the sign ambiguity in $\beta$. Both of $J$ and $\sin (2 \beta)$ are slightly smaller then the experimental values. However, since this is only an illustrative configuration, and there may be others that work as well, the significance is not in exact agreement, but rather 
in the fact that they are very close. We note for completeness that this configuration predicts $\alpha=2.37$ and $\gamma=0.430$.

There are a few points to note about this configuration. First, the largest ratio of widths is $9.865 / 2.365 \approx 4$, so the widths of all the fermion fields are of the same order of magnitude, with $\mathcal{O}(1)$ differences between them. Second, the phases required are very small. That small phases are required can be easily understood by noting that the mechanism for generating the hierarchy depends on the singularity of the Yukawa matrices. A large phase would ruin that singularity. It is thus significant that these very small phases are enough to generate the observed CP-violation.

\section{Flavor Changing Processes}

The above discussion of the generation of the Yukawa hierarchy made no mention of the overall scale of the fermion widths. This is can be understood from the fact that the Yukawa couplings in Eq. (2.4) involve only ratios of widths, and hence are independent of any overall scale.

Effects that depend on the scale will come from interactions with the Kaluza-Klein (KK) excitations of the gauge bosons. These will turn out to depend explicitly on $R$, as well as on the ratio $\rho=\sigma / R$. Here $\sigma$ is taken to be the generic scale of the fermion widths, with $\sigma_{i}=\gamma_{i} \sigma$ where $\gamma_{i}$ is of order unity, and we further define $\rho_{i}=\sigma_{i} / R$ for later convenience. We see that $\rho$ is a measure of the separation between the energy scales of the fermions (the localization energy) and the bosons (the compactification scale).

For simplicity at this point we take the extra dimension to be flat. The bosons are allowed to propagate in the entire bulk, which has size $R$. The wavefunctions are then

$$
A^{(n)}(y)=\frac{1}{\sqrt{R}} e^{\frac{i n \pi y}{R}}
$$

In most models half of these will be projected out by boundary conditions. We retain only the cosine modes, to preserve the zero modes $(n=0)$ which correspond to the Standard Model bosons. The gauge couplings are then

$$
\begin{aligned}
g_{i}^{(n)} & =g_{5} \int_{0}^{R} d y A^{(n)}(y) \bar{\psi}_{i}(y) \psi_{i}(y) \\
& =\sqrt{2} g_{4} \frac{\left[1-e^{-\frac{1}{\rho_{i}}}\right]}{1+\frac{n^{2} \pi^{2} \rho_{i}^{2}}{4}}, \quad n \geq 1,
\end{aligned}
$$


for $a=1$, and

$$
g_{i}^{(n)}=\sqrt{2} g_{4} e^{-\frac{1}{8} n^{2} \rho_{i}^{2}}, \quad n \geq 1
$$

for $a=2$.

These couplings depend on the fermion species. To see that this results in flavorchanging currents, consider the phenomenological 4D Lagrangian for the quarks, $\boldsymbol{d}_{L}=$ $\left(d_{L}, s_{L}, b_{L}\right)^{\dagger}$ and similarly for $\mathbf{d}_{R}, \mathbf{u}_{L}, \mathbf{u}_{R}$, all coupled to the gluon field $G$ :

$$
\begin{aligned}
\mathcal{L}= & \mathcal{L}_{\text {kinetic }}+g G_{\mu}^{0} \sum_{i=L, R}\left(\overline{\boldsymbol{d}}_{i} \gamma^{\mu} \boldsymbol{d}_{i}+\overline{\boldsymbol{u}}_{i} \gamma^{\mu} \boldsymbol{u}_{i}\right)+\sqrt{2} g \sum_{n=1}^{\infty} G_{\mu}^{(n)} \sum_{i=L, R}\left(\overline{\boldsymbol{d}}_{i} C_{i}^{d(n)} \gamma^{\mu} \boldsymbol{d}_{i}+\overline{\boldsymbol{u}}_{i} C_{i}^{u(n)} \gamma^{\mu} \boldsymbol{u}_{i}\right) \\
& +\boldsymbol{d}_{L} V_{L}^{d \dagger} M_{d} V_{R}^{d} \boldsymbol{d}_{R}+\boldsymbol{u}_{L} V_{L}^{u \dagger} M_{u} V_{R}^{u} \boldsymbol{u}_{R}
\end{aligned}
$$

where $M_{u, d}$ are the diagonal mass matrices, the $V_{L, R}^{(u, d)}$ are the unitary matrices that accomplish the diagonalization, the $G^{(n)}$ are the Kaluza-Klein excitations of the gluons, and we have ignored all but the zero modes for the fermions, since the fermion KK modes will not enter the processes considered here. The couplings to the KK gluons are contained in the diagonal matrices $C_{L, R}^{u, d(n)}$, with the $i$-th coupling given by Eqs. (4.2) and (4.3). When we transform to the mass basis the gauge couplings will be the elements of the matrices

$$
U_{L, R}^{u, d(n)} \equiv V_{L, R}^{u, d \dagger} C_{L, R}^{u, d(n)} V_{L, R}^{u, d}
$$

Since the $C$ matrices are not the identity, these couplings are not diagonal in flavor space, and hence there will be flavor-changing currents in the KK gauge sector. In particular there will be tree-level FCNC mediated by the KK gluon states.

Processes that involve these tree-level FCNC are the source of the strong constraints on split fermion models. However, if one compares the FCNC effects in our model to the split fermion models, for the same value of the $\rho$ parameter, the magnitude turns out to be much smaller. We can measure the magnitude of the FCNC with the difference of couplings between two fermion species $g_{i}^{(n)}-g_{j}^{(n)}$, since the FCNC will vanish if this difference does. Figure 3 shows the difference of couplings as a function of the KK number $n$, of two fermion species; in the first case two fermions of the same width and $\rho=1 / 10$ separated by $4 \sigma$; in the second two fermions at the same location, one with $\rho_{i}=1 / 10$, the other with $\rho_{j}=1 / 20$. The total effect is the sum over $n$, or roughly the integral of the curves. Clearly the effect is much smaller in the variable width case.

The reason for this suppression can be understood quite simply. When the fermion species are directly on top of one another and with the same width the flavor changing currents are zero. When they are separated the non-universality of the couplings comes 

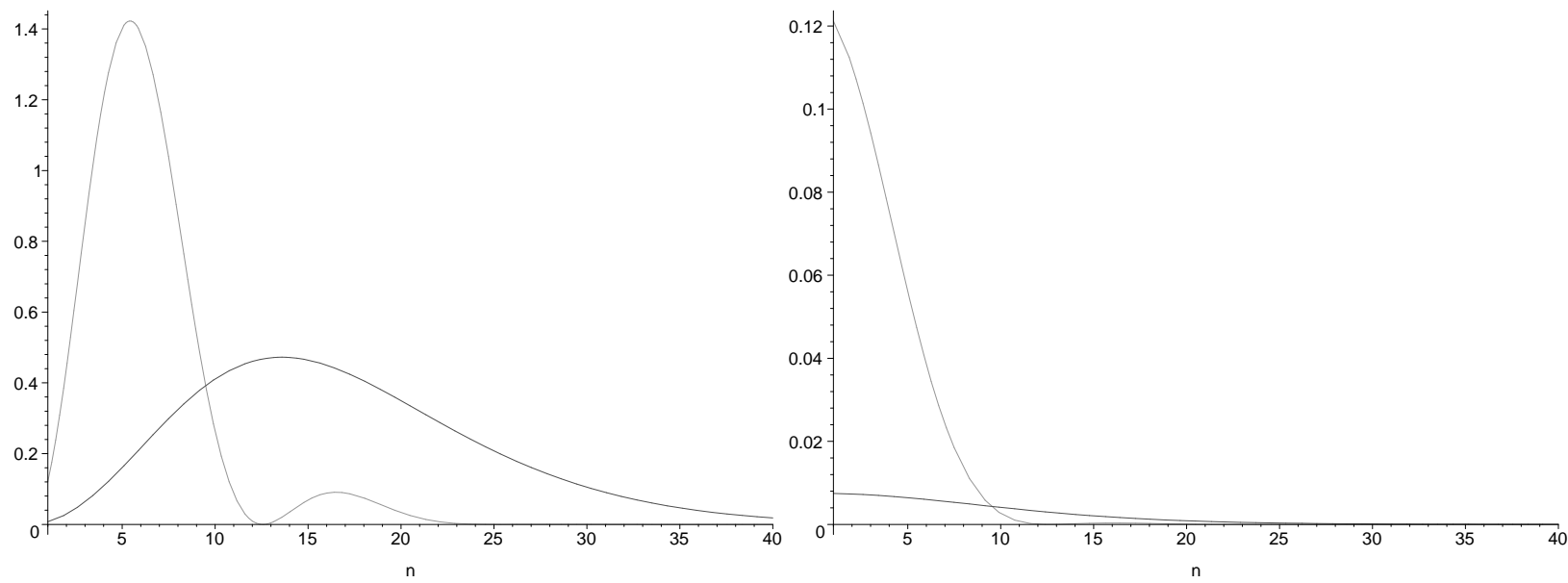

Figure 3: Comparison of the flavor-changing effects of models with split fermions (upper curve) and different widths with $a=2$ (lower), for the same hierarchy of scales, $\sigma / R=1 / 10$. Left: the difference of couplings as a function of $n$. Right: the same difference multiplied by $1 / n^{2}$, as it would appear in the KK sum. Flavor changing currents are proportional to the sum over $n$ of the right curves.

from the different heights of the KK wavefunctions at the location of the fermions. So every KK state, starting from $n=1$, can resolve the difference, up to the cutoff $n \approx 1 / \rho$. When the fermions are localized to the same point and the widths are changed the non-universality can only start to be resolved when the KK wavefunctions oscillate fast enough to resolve the widths. In this case the first few KK states will have nearly universal couplings, and the large flavor difference won't start until roughly $n \approx 1 / \rho_{>}$, where $\rho_{>}$is the larger of the two widths, and will only last until $n \approx 1 / \rho_{<}$. Additionally, if there is only one extra dimension, the mass in the $\mathrm{KK}$ propagator provides a $1 / n^{2}$ suppression in the sum, further suppressing the FCNC from variable widths, while leaving the FCNC from the first few KK modes from split fermions large. Note that this argument does not depend crucially on the shape of the fermion wavefunctions. The key points are the slow variation of the gauge wavefunctions for small $n$ over the size of the fermions, and the $1 / n^{2}$ suppression in the propagator. Thus the argument holds for any value of $a$, and indeed would hold for very different, potentially very irregular, shapes of the fermions. The only requirement is that they all fall off exponentially or faster from the same point on length scales of order the first KK mass.

The most stringent specific constraint comes from the mass splitting in the neutral kaon sector, $\Delta m_{K}$. The calculation of $\Delta m_{K}$ here is identical with that in the split 
fermion case, except for the form of the sum over KK-modes. In [32] it was found that

$$
\begin{array}{rl}
\Delta m_{K}= & \operatorname{Re}\left\langle\bar{K}^{0}\left|\mathcal{L}^{\Delta S=2}\right| K^{0}\right\rangle \\
=\frac{2}{3} R^{2} f_{K}^{2} m_{K} & \mathcal{R} e\left(\left|V_{L 11}^{d} V_{L 12}^{d *}\right|^{2} \zeta_{1} \sum_{n=1}^{\infty} \frac{\left(g_{d_{L}}-g_{s_{L}}\right)^{2}}{n^{2}}+\left|V_{R}^{d}{ }_{11} V_{R}^{d *}{ }_{12}\right|^{2} \zeta_{1} \sum_{n=1}^{\infty} \frac{\left(g_{d_{R}}-g_{s_{R}}\right)^{2}}{n^{2}}\right. \\
& +\left(V_{L 11}^{d} V_{L 12}^{d *} V_{R}^{d *}{ }_{11} V_{R}^{d}{ }_{12}\right) \zeta_{2} \sum_{n=1}^{\infty} \frac{\left(g_{d_{L}}-g_{s_{L}}\right)\left(g_{d_{R}}-g_{s_{R}}\right)}{n^{2}} \\
& \left.+\left(V_{R 11}^{d} V_{R}^{* d}{ }_{12} V_{L}^{* d}{ }_{11} V_{L}^{d}{ }_{12}\right) \zeta_{2} \sum_{n=1}^{\infty} \frac{\left(g_{d_{L}}-g_{s_{L}}\right)\left(g_{d_{R}}-g_{s_{R}}\right)}{n^{2}}\right)
\end{array}
$$

where the $\zeta_{i}$ are the dimensionless part of the hadronic matrix elements and are given by

$$
\begin{aligned}
\zeta_{1} & =\frac{1}{3}, \\
\zeta_{2} & =\left(\frac{1}{12}+\frac{1}{4}\left(\frac{m_{K}^{2}}{m_{d}^{2}+m_{s}^{2}}\right)\right),
\end{aligned}
$$

when computed in the vacuum insertion approximation [37. Requiring this to not be larger than the observed value produces a constraint on $1 / R$, given a value of $\rho$,

$$
\frac{1}{R} \geq 1960 \mathrm{TeV} \sqrt{\mathcal{R} e\left(\zeta_{1} \sum(\mathrm{LL}+\mathrm{RR})+\zeta_{2} \sum(\mathrm{LR}+\mathrm{RL})\right)},
$$

where LL is the left-left term in Eq. (4.6), etc..

We have calculated $\Delta m_{K}$ for the configuration given in section 3. Fig. 4 shows the constraint for a range of $\rho$. This demonstrates clear power-law behavior, with $1 / R=(73.8 \mathrm{TeV}) \rho^{1 / 2}$. We see that for very large values, say $\rho=10^{-1}$, we have large constraints $1 / R \geq 30 \mathrm{TeV}$. One can get down to the direct production constraint of $1 / R \geq 2 \mathrm{TeV}$ by going to $\rho \approx 10^{-3}$. The constraints here turn out to be very close to those obtained for the model of Kaplan and Tait [22], where they localized fermions to exponentials centered at one of two fixed points, much like here, but still produced small Yukawa matrix elements by separating the left and right handed components. ${ }^{1}$ Since they presumably have very different $V_{L, R}^{u, d}$ mixing elements, this shows that the flavor constraints on variable width models are quite robust. These constraints are to be contrasted with the similar results for the split fermion case, where $1 / R \geq 400 \mathrm{TeV}$ for $\rho=10^{-1}$ and $1 / R \geq 60 \mathrm{TeV}$ for $\rho=10^{-3}$; in those models one must go to $\rho \approx 10^{-5}$ before the flavor constraints are similar to direct production constraints.

${ }^{1}$ The paper [22] presents results for $\rho=10^{-1}$. The apparent disagreement between the numbers quoted there and here is due to a normalization factor of $2 \pi$ between their $M_{c}$ and our $1 / R$. 


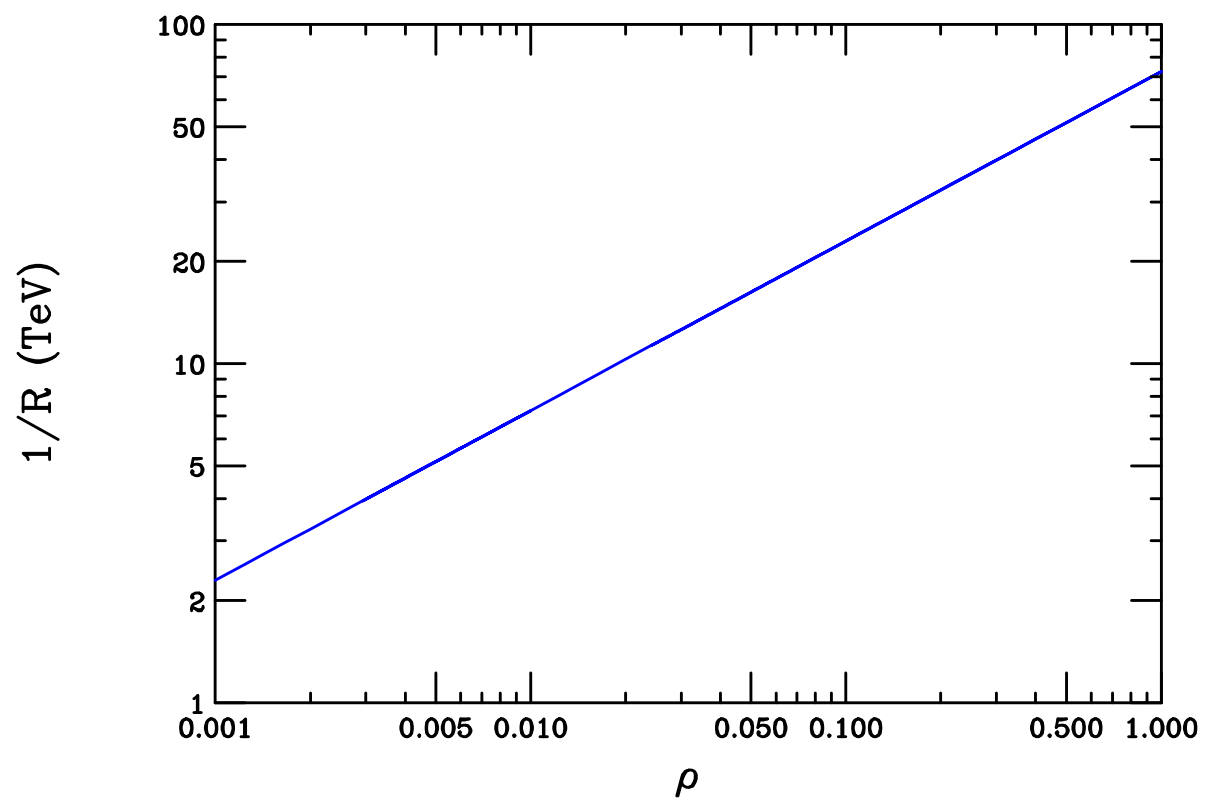

Figure 4: Constraints on the compactification scale $1 / R$ from $\Delta m_{K}$ as a function of $\rho=\sigma / R$, the ratio of the compactification scale to the fermion localization scale. The area below the curve is excluded.

Note that in several places it was claimed that the constraint from $\epsilon_{K}$ was even larger than that from $\Delta m_{K}$ [30, 31, 22]. This can be seen from the $\epsilon_{K}$ equivalent to Eq. (4.8)

$$
\frac{1}{R} \geq 40,900 \mathrm{TeV} \sqrt{\operatorname{Im}\left(\zeta_{1} \sum(\mathrm{LL}+\mathrm{RR})+\zeta_{2} \sum(\mathrm{LR}+\mathrm{RL})\right)}
$$

However, the imaginary part is, in fact, quite small. For $\rho=10^{-1}$, the factor in the square root is $1.6 \times 10^{-3}$, leading to a constraint $1 / R \geq 6.6 \mathrm{TeV}$; this is much smaller than the constraint from $\Delta m_{K}$.

\section{Discussion}

In previous sections we have tried to emphasize the aspects which are independent of the mechanism for the variable width scenario of fermion localization. Here we discuss possible techniques of fermion localization that produce this scenario. The easiest implementation of localized fermions is on a five-dimensional space with the extra dimension compactified on $S_{1} / Z_{2}$. The fermions are then coupled to a scalar field that is odd under the $Z_{2}$ action of the orbifold. It can then be shown that the fermions 
will develop chiral zero modes localized near one of the orbifold fixed points 38]. This Lagrangian is given by

$$
\mathcal{L}=\sum_{i} \bar{\Psi}_{i}\left(i \not \partial-\gamma^{5} \partial_{5}-f_{i} \varphi\right) \Psi_{i}+\frac{1}{2} \partial^{\mu} \varphi \partial_{\mu} \varphi-\frac{1}{2} \partial_{5} \varphi \partial_{5} \varphi-\frac{\lambda}{4}\left(\varphi^{2}-v^{2}\right)^{2},
$$

where here we allow each fermion to have a separate coupling, $f_{i}$, to $\varphi$. Since $\varphi$ is odd under the orbifolding it must vanish at each of the fixed points, $y=0$ and $y=\pi R$. However, if $\lambda v^{2}$ is large enough $\varphi$ will develop a $y$-dependent vev, $h(y)$, that is zero on the fixed points and is non-vanishing elsewhere. The fermions then develop zero modes with profiles

$$
\psi_{i}(y) \propto e^{-f_{i} \int_{0}^{y} d y^{\prime} h\left(y^{\prime}\right)} .
$$

This is localized near $y=0$ if $f_{i} h(y)>0$, and at $y=R$ otherwise. The class of models parameterized by $a$ in Eq. (2.1) can be constructed by demanding that $h(y)$ behave like $h(y) \approx k y^{a-1}$ near $y=0$ for some constant $k$, and picking all the $f$ to have the same sign as $h(y)$, so all fermions are localized to $y=0$. The fermions are then localized as in Eq. (2.1) with width parameter $\sigma_{i}=\left(f_{i} k\right)^{-1 / a}$.

Note that this construction does not appear to generate any non-trivial phases in the Yukawa matrices with which we could generate $\phi_{1,2}$. However, phases will be present if one allows the localizer field $\varphi$ to be complex and imposing boundary conditions on the phase at the orbifold fixed points. For instance we could require that $\arg (\varphi(y=0))=0$ and $\arg (\varphi(y=R))=\pi / 2$. The vev then has a profile $v e^{i \frac{\pi}{2} \frac{y}{R}}$. This still results in localized fermions, but the Yukawa matrix elements pick up phases relative to each other, since the phase at each point will be weighted differently due to the distinct widths. For fermions with width ratios of order those given in the solution in section 3 with $1 / R \approx 5 \mathrm{TeV}$, we find phase differences of order $\phi=0.03$, so this looks like a promising way to make a realistic construction of this scenario.

Another interesting example of fermion localization occurs in the RS scenario. In this case, the fermions are localized near a brane with $a=1$ profiles, and possibly different widths [39]. The gauge boson wavefunctions are Bessel functions rather than cosines, so the flavor analysis in section 4 is not strictly applicable. However, since the low KK-number wavefunctions are reasonably flat, the reasoning that the flavor changing currents are small will still hold. This has been seen explicitly in 33.

One would like to embed the variable widths model in a larger scenario that, for instance, solves the Higgs mass hierarchy problem. The fermions and gauge bosons could be localized within a thick brane embedded in a larger dimension of the ADD type. Or the complete manifold could be $A d S_{5} \times\left(S_{1} / Z_{2}\right)$ with the RS scenario playing out in 


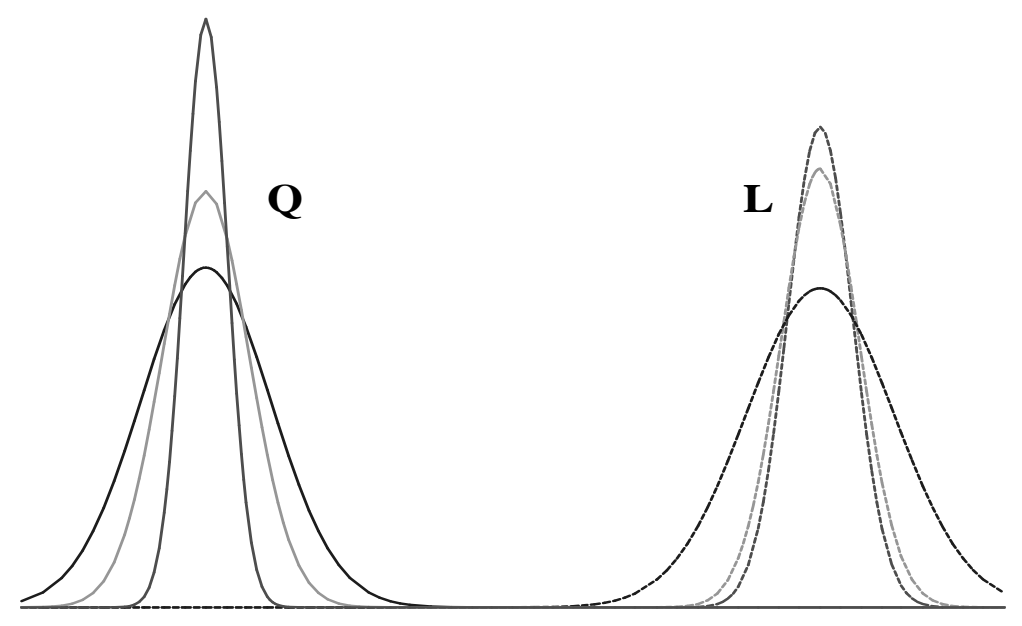

Figure 5: Illustration of a configuration that would suppress proton decay while generating the Yukawa hierarchy with variable widths.

the $A d S_{5}$ and the fermion mass generation playing out in the $S_{1} / Z_{2}$ 40. Unfortunately, with all fermions localized at a single point there is nothing to suppress the proton decay operators that tend to occur in these models, unlike in the original split fermion scenario. However, a simple twist on this scenario can restore this suppression. Instead of a single fixed point, there could be two points, with the quark fields localized on one of them, and the leptons on the other, as shown in Fig. 5. Proton decay operators are then suppressed by approximately $e^{-\frac{3}{4} l^{2}}$, where $l$ is the separation between the two fixed points, in units of the scale of the fermion widths. Note that this changes none of the conclusions above about the generation of the mass hierarchy. In the simple $S_{1} / Z_{2}$ model presented above this could be accomplished by taking the couplings to $\varphi$ for quarks to be $f_{i}>0$ and for leptons $f_{i}<0$.

We could also try to modify the model in order to generate the ratios $\lambda_{5}^{u} / \lambda_{5}^{d}$ and $\lambda_{5}^{u} / \lambda_{5}^{e}$, rather than having to put them in by hand. One way to do this would be to make use of the original AS suggestion of separating left and right handed fermions. The up-type singlets, $u_{i}$, could be very close to the quark doublets, $Q_{i}$. The down-type singlets, $d_{i}$, could then be farther away (about $2 \sigma$ for $a=2$ ), and the lepton doublets and charged singlets separated slightly farther than that. This is then a hybrid model where the hierarchies between fermions with the same charge are generated by the different widths, and the hierarchy between those with different charges is generated by the exponentially small overlaps. Combined with separating the lepton wavefunctions to suppress proton decay, one obtains the picture in Fig. 6. The whole assembly with $a=2$ requires about $10 \sigma$ of length in the additional dimension; most of that is needed 


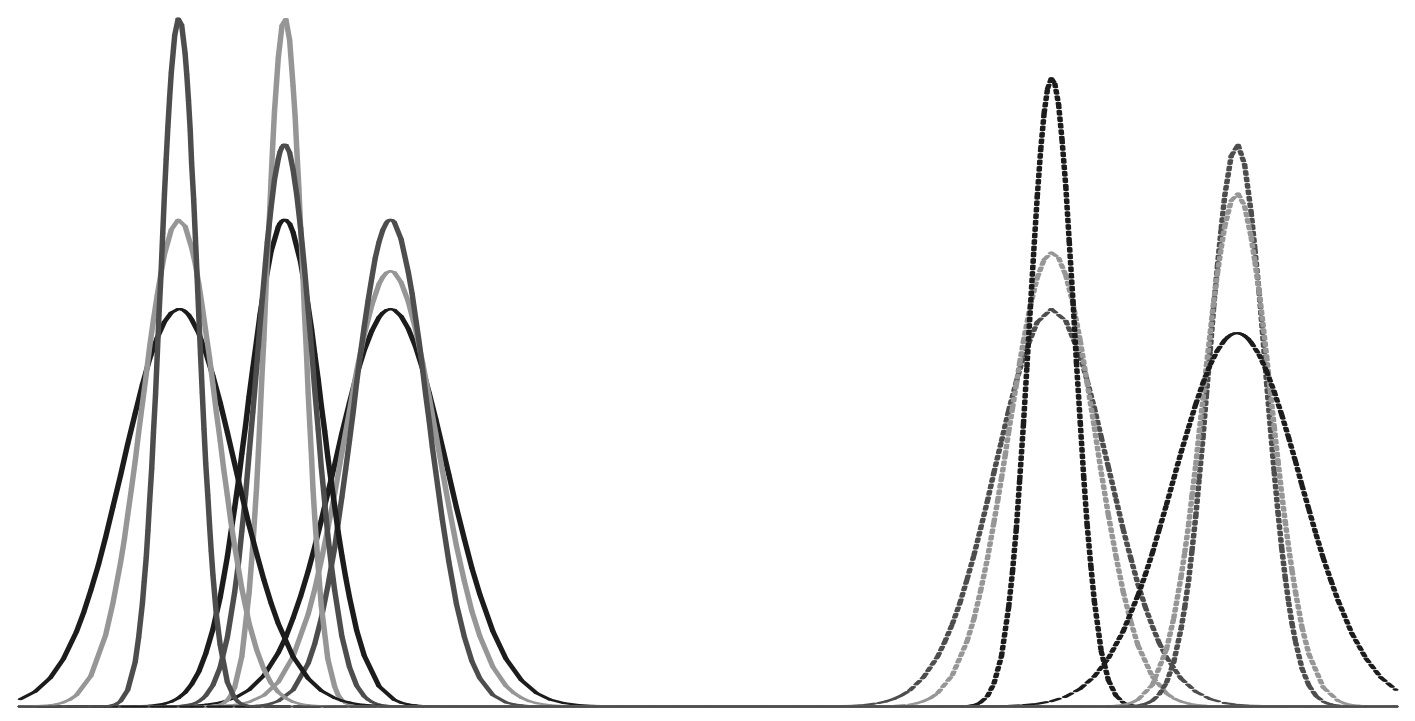

Figure 6: Illustration showing a "Swiss Army Knife" configuration. It generates the hierarchy between generations with the variable width method; uses split fermions to generate the hierarchy between the top mass and the bottom and $\tau$ masses; and suppresses proton decay by localizing quarks and fermions to different fixed points.

to suppress proton decay. Note that as long as all fermions with the same quantum numbers are localized to the same place the suppression of FCNC described above will apply.

\section{Conclusion}

We have proposed a model that localizes fermions with different widths in a single, compact, extra dimensional bulk, and shown that this produces a realization of the democratic scenario of fermion mass matrices. Ratios of the fermions widths that are $\mathcal{O}(1)$ can produce the observed fermion masses and mixing angles. With the inclusion of additional small phases one can reproduce all Standard Model parameters in the fermion sector, including the CP-violating CKM phase. This is not a reduction of parameters, but it does explain the large hierarchy in Yukawa couplings in terms of a simple physical picture. The tree-level FCNC contributions are smaller than in similar models by about a factor of 10 per flavor changing vertex. The resulting constraints on the compactification scale can be as small as $1-2 \mathrm{TeV}$ if one allows the ratio of localization to compactification scales to be $\rho \approx 10^{-3}$. This advantage in reducing FCNC effects comes not so much from the particular mechanism of localization, but rather from the facts that all fermions are localized to the same point, and that the gauge boson 
KK wavefunctions are very smooth for the first few modes, so they fail to resolve the difference in fermion zero modes. When the KK mode number $n$ is high enough to resolve the differences in fermions the $1 / n^{2}$ suppression is large. It is also interesting that the mechanism for generating the Yukawa hierarchy does not depend on the shape of the fermion wavefunction near the localization point, as long as it is exponential far away. It only requires $\mathcal{O}(1)$ differences between overlaps of the wavefunctions. As long as they were all appropriately localized, the zero-mode wavefunctions could be extremely irregular and still generate the correct fermion hierarchy while being consistent with the FCNC constraints. It may also be possible to reduce the FCNC constraints further by the inclusion of the effects of brane-localized kinetic terms, which were not considered here 41, 42].

Effects of variable width localization could be observable at future colliders, particularly in flavor-changing processes. Even if direct effects are not seen at the LHC, precision measurements of mass splittings or rare $B$ decays may provide clues. In [28], it was noted that when fermions are separated in an extra dimension, one might be able to observe cross sections that fall exponentially in the center-of-mass energy in certain channels. There it was proposed to look at polarized $e^{+} e^{-}$collisions to search for split fermions. In the scenario presented here this may apply to high energy ep collisions if the quark and lepton separation prevents proton decay.

Note Added: While this paper was being completed a similar paper [43] appeared. There, a specific model for the vev in section 5 was made and the Standard Model parameters were obtained.

\section{Acknowledgments}

The author would like to thank Tom Rizzo, Frank Petriello, JoAnne Hewett, and Michael Peskin for many useful discussions.

\section{References}

[1] I. Antoniadis, A possible new dimension at a few tev, Phys. Lett. B246 (1990) 377-384.

[2] J. D. Lykken, Weak scale superstrings, Phys. Rev. D54 (1996) 3693-3697, hep-th/9603133.

[3] E. Witten, Strong coupling expansion of calabi-yau compactification, Nucl. Phys. B471 (1996) 135-158, hep-th/9602070. 
[4] P. Horava and E. Witten, Eleven-dimensional supergravity on a manifold with boundary, Nucl. Phys. B475 (1996) 94-114, hep-th/9603142].

[5] P. Horava and E. Witten, Heterotic and type i string dynamics from eleven dimensions, Nucl. Phys. B460 (1996) 506-524, hep-th/9510209.

[6] E. Caceres, V. S. Kaplunovsky, and I. M. Mandelberg, Large-volume string compactifications, revisited, Nucl. Phys. B493 (1997) 73-100, hep-th/9606036.

[7] N. Arkani-Hamed, S. Dimopoulos, and G. R. Dvali, The hierarchy problem and new dimensions at a millimeter, Phys. Lett. B429 (1998) 263-272, hep-ph/9803315.

[8] I. Antoniadis, N. Arkani-Hamed, S. Dimopoulos, and G. R. Dvali, New dimensions at a millimeter to a fermi and superstrings at a tev, Phys. Lett. B436 (1998) 257-263, hep-ph/9804398.

[9] N. Arkani-Hamed, S. Dimopoulos, and G. R. Dvali, Phenomenology, astrophysics and cosmology of theories with sub-millimeter dimensions and tev scale quantum gravity, Phys. Rev. D59 (1999) 086004, hep-ph/9807344.

[10] L. Randall and R. Sundrum, A large mass hierarchy from a small extra dimension, Phys. Rev. Lett. 83 (1999) 3370-3373, hep-ph/9905221.

[11] L. Randall and R. Sundrum, An alternative to compactification, Phys. Rev. Lett. 83 (1999) 4690-4693, hep-th/9906064.

[12] B. A. Dobrescu and E. Poppitz, Number of fermion generations derived from anomaly cancellation, Phys. Rev. Lett. 87 (2001) 031801, hep-ph/0102010.

[13] T. Appelquist, H.-C. Cheng, and B. A. Dobrescu, Bounds on universal extra dimensions, Phys. Rev. D64 (2001) 035002, hep-ph/0012100.

[14] G. Servant and T. M. P. Tait, Is the lightest kaluza-klein particle a viable dark matter candidate?, Nucl. Phys. B650 (2003) 391-419, hep-ph/0206071.

[15] G. Servant and T. M. P. Tait, Elastic scattering and direct detection of kaluza-klein dark matter, New J. Phys. 4 (2002) 99, hep-ph/0209262.

[16] H.-C. Cheng, J. L. Feng, and K. T. Matchev, Kaluza-klein dark matter, Phys. Rev. Lett. 89 (2002) 211301, hep-ph/0207125.

[17] N. Arkani-Hamed and M. Schmaltz, Hierarchies without symmetries from extra dimensions, Phys. Rev. D61 (2000) 033005, hep-ph/9903417.

[18] Y. Grossman and G. Perez, Realistic construction of split fermion models, Phys. Rev. D67 (2003) 015011, hep-ph/0210053. 
[19] E. A. Mirabelli and M. Schmaltz, Yukawa hierarchies from split fermions in extra dimensions, Phys. Rev. D61 (2000) 113011, hep-ph/9912265.

[20] W.-F. Chang and J. N. Ng, Cp violation in 5d split fermions scenario, JHEP 12 (2002) 077, hep-ph/0210414.

[21] F. Del Aguila and J. Santiago, Signals from extra dimensions decoupled from the compactification scale, JHEP 03 (2002) 010, hep-ph/0111047.

[22] D. E. Kaplan and T. M. P. Tait, New tools for fermion masses from extra dimensions, JHEP 11 (2001) 051, hep-ph/0110126.

[23] G. C. Branco, A. de Gouvea, and M. N. Rebelo, Split fermions in extra dimensions and cp violation, Phys. Lett. B506 (2001) 115-122, hep-ph/0012289.

[24] T. G. Rizzo and J. D. Wells, Electroweak precision measurements and collider probes of the standard model with large extra dimensions, Phys. Rev. D61 (2000) 016007, hep-ph/9906234.

[25] M. Masip and A. Pomarol, Effects of sm kaluza-klein excitations on electroweak observables, Phys. Rev. D60 (1999) 096005, hep-ph/9902467.

[26] W. J. Marciano, Fermi constants and 'new physics', Phys. Rev. D60 (1999) 093006, hep-ph/9903451.

[27] J. Hewett and M. Spiropulu, Particle physics probes of extra spacetime dimensions, Ann. Rev. Nucl. Part. Sci. 52 (2002) 397-424, hep-ph/0205106.

[28] N. Arkani-Hamed, Y. Grossman, and M. Schmaltz, Split fermions in extra dimensions and exponentially small cross-sections at future colliders, Phys. Rev. D61 (2000) 115004, hep-ph/9909411.

[29] T. G. Rizzo, Cartography with accelerators: Locating fermions in extra dimensions at future lepton colliders, Phys. Rev. D64 (2001) 015003, hep-ph/0101278.

[30] A. Delgado, A. Pomarol, and M. Quiros, Electroweak and flavor physics in extensions of the standard model with large extra dimensions, JHEP 01 (2000) 030, hep-ph/9911252.

[31] S. A. Abel, M. Masip, and J. Santiago, Flavour changing neutral currents in intersecting brane models, hep-ph/0303087.

[32] B. Lillie and J. Hewett, Flavor constraints on split fermion models, hep-ph/0306193.

[33] S. J. Huber, Flavor violation and warped geometry, hep-ph/0303183. 
[34] H. Fritzsch and Z.-z. Xing, Mass and flavor mixing schemes of quarks and leptons, Prog. Part. Nucl. Phys. 45 (2000) 1-81, hep-ph/9912358.

[35] Particle Data Group Collaboration, K. Hagiwara et. al., Review of particle physics, Phys. Rev. D66 (2002) 010001.

[36] G. Raven, sin(2beta): Status and prospects, hep-ex/0307067.

[37] F. Gabbiani, E. Gabrielli, A. Masiero, and L. Silvestrini, A complete analysis of fcnc and cp constraints in general susy extensions of the standard model, Nucl. Phys. B477 (1996) 321-352, hep-ph/9604387.

[38] H. Georgi, A. K. Grant, and G. Hailu, Chiral fermions, orbifolds, scalars and fat branes, Phys. Rev. D63 (2001) 064027, hep-ph/0007350.

[39] J. L. Hewett, F. J. Petriello, and T. G. Rizzo, Precision measurements and fermion geography in the randall-sundrum model revisited, JHEP 09 (2002) 030, hep-ph/0203091.

[40] H. Davoudiasl, J. L. Hewett, and T. G. Rizzo, Phenomenology on a slice of ads(5) $x$ $m^{* *}$ delta spacetime, JHEP 04 (2003) 001, hep-ph/0211377.

[41] M. Carena, T. M. P. Tait, and C. E. M. Wagner, Branes and orbifolds are opaque, Acta Phys. Polon. B33 (2002) 2355, hep-ph/0207056.

[42] F. del Aguila, M. Perez-Victoria, and J. Santiago, Bulk fields with general brane kinetic terms, JHEP 02 (2003) 051, hep-th/0302023.

[43] A. Soddu and N.-K. Tran, Democratic mass matrices from five dimensions, hep-ph/0308043. 